\title{
Angst als mangelnde Hoffnung. Marianne Grubers Erinnerungen eines Narren (2012)
}

Fear as a lack of hope. Marianne Gruber's Memoirs of a Fool (2012)

Václava Beyerová

\begin{abstract}
In Marianne Gruber's latest novel Erinnerungen eines Narren (2012), the theme of fear appears in various forms - first as a part of life and later as an inexplicable fear. This paper examines the forms of fear in Marianne Gruber's novel, basing the research on Sigmund Freud's conceptualization of fear. Another aim of the paper is to find out how dealing with fear is treated in literature.
\end{abstract}

\section{Keywords}

Fear; Marianne Gruber; trauma; Sigmund Freud; Albert Camus; meaning of life 


\section{Einleitung}

Marianne Gruber greift in ihrem letzten Roman Erinnerungen eines Narren einen existentiellen Stoff auf - wie man Angst durch Erzählungen besänftigt. Der Protagonist nennt sich „einen geschwätziger Erzähler des Schweigens, [...] man findet anders seinen Frieden nicht." ${ }^{1}$ Es handelt sich bei ihm um einen Menschen, der nach dem Sturz vom Hochseil im Bett liegt und sich nicht mehr bewegen kann. Seine Adressatin ist wohl eine Frau, die ihm recht nahe steht und seine Nachfolge als Clown antreten sollte. Im Kopf kreisen ihm chaotisch viele Geschichten. Diese Gedankengänge sind voll von Sprüngen, die seltsam sind, und doch durch die Erzählerfigur des Clowns zusammenhängen. Protokolle der Angst hieß Marianne Grubers zweites Buch, ein Erzählband aus dem Jahr 1983. Das Motiv der Angst ist ein Gegenpol zur ebenso irrational erlebten Hoffnung. Die Angst wird dadurch überwunden, dass man versucht mit der Welt Frieden zu schließen und schweigt.

Das Ziel dieses Beitrags ist eine etwas andere Lesart des Romans vorzulegen als die von Evelyne Polt-Heinzl, die ihn „ein[en] Kampf um Würde und um Akzeptanz des Unausweichlichen" ${ }^{2}$ nannte. Können die in der Psychologie entwickelten Erkenntnisse über die Angst auf einen literarischen Text sinnvoll angewendet werden? Oder ist es - im Gegenteil - ein weiterer Umgang mit dem Sisyphos-Stoff?

\section{Angst als anthropologische Größe}

Angst ist ein grundsätzlicher Bestandteil menschlicher Existenz, der in der Psychologie auch empirisch erforscht wird. Dieser Untersuchungsgegenstand ist zwar in der Psychologie verankert, es gibt aber immer ,offene" Themen zur Untersuchung. Den theoretischen Rahmen für die Analyse dieses Romans bildet Sigmund Freuds Konzept der Angst. Sigmund Freud beschäftigte sich wie Viktor Frankl mit dem Wesen und der Behandlung von Neurosen. Freud fand ihre Ursachen in der Angst, die durch widersprüchliche und unbewusste Motive ausgelöst wird. Frankl unterscheidet mehrere Formen von Neurosen, von denen er mehrere auf die Unfähigkeit des Patienten zurückführte, einen Sinn in seiner Existenz zu finden. In Europa ist gegenwärtig eine Abkehr von Freud und stattdessen ein Interesse an der Übernahme der Existenztheorie zu beobachten, die zum Beispiel in der Logotherapie zum Ausdruck kommt. Frankl lehnt Freud nicht ab, sondern baut auf dessen Forschungen auf. ${ }^{3}$

Frankls Suche nach dem Sinn des Lebens hat einen starken Einfluss auf Marianne Gruber ausgeübt. ${ }^{4}$ Gruber behauptet, dass vorformulierte Aussagen, wie Freuds Men-

1 Gruber, Marianne (2012): Erinnerungen eines Narren. Innsbruck - Wien: Haymon, S. 295.

2 Vgl. Polt-Heinzl, Evelyne (2012): Der Clown als stummer Zeuge. In: Die Presse, 15.06.2012 [online]. [Zit. 15.11.2021]. Erreichbar unter: https://www.diepresse.com/766285/der-clown-als-stummer-zeuge

3 Frankl, Viktor E. (1992): Man's Search for Meaning. An introduction to logotherapy. Fourth Edition, S. 8-9.

4 Vgl. Weidenhiller, Ute (1995): Ein Gespräch mit Marianne Gruber am 8. August 1995 in Steindorf am 
schenbild aus der Psychologie oder Jungs Archetypen, in die künstlerische Sphäre übernommen werden, ${ }^{5}$ was bedeutet, dass die Kunst mit der Psychologie verbunden ist. Das Ergebnis kann gerade eine psychologisch motivierte Interpretation eines literarischen Textes sein.

\subsection{Angst als Signal}

Freuds Angst-Forschungen waren zuerst eher klinischer Natur. 1894 schrieb er Über die Berechtigung, von der Neurasthenie einen bestimmten Symptomenkomplex als "Angst-Neurose“ abzutrennen. ${ }^{6}$ Darin wies er auf seine eigene Untersuchung der seelischen Krankheiten hin. In Freuds Forschungsschriften unterscheidet man die erste und die zweite Theorie der Angst. Freuds frühere Arbeiten erklären die Angstneurose im Zusammenhang mit der Hysterie und sind für unsere Fragestellung weniger relevant. ${ }^{7}$ Folgende Formulierung stammt aus der zweiten Angsttheorie, die Angst als Signal deutet. Freud stützte sich auf psychoanalytische Affekttheorien, weil die Angst ein „Affektzustand“8 ist. Er untersucht Affekt im Zusammenhang mit Trieb, und zwar beschreibt er drei Reaktionen, die der Trieb auslösen kann:

Der Trieb wird entweder ganz unterdrückt, so dass man nichts von ihm auffindet, oder er kommt als irgendwie qualitativ gefärbter Affekt zum Vorschein, oder er wird in Angst verwandelt. Die beiden letzteren Möglichkeiten stellen uns die Aufgabe, die Umsetzung der psychischen Energien der Triebe in Affekte und ganz besonders in Angst als neues Triebschicksal ins Auge zu fassen. ${ }^{9}$

Bei seinen klinischen Untersuchungen hat Freud festgestellt, dass im Fall der Angstneurose Störungen in der Abfuhr der Sexualspannung vorkommen. Er behauptet, dass die angehäufte Erregung sich nach außen in Angst umwandelt oder umzuwandeln versucht.

Ossiachersee in Marianne Gruber und die österreichische Gegenwartsliteratur. Die Wille zum Sinn in einer sinnlosen Welt, 1995, S. 34.

5 Vgl. Gruber, Marianne (1977): Existenzanalyse und Literatur. In: Podium 26, S. 19-23.

6 Freud, Sigmund (1999): Gesammelte Werke. Bd. I. Frankfurt am Main: Fischer, S. 313- 342.

7 Die Psyche gerät in den Affekt der Angst, wenn sie sich unfähig fühlt, eine von außen nahende Aufgabe (Gefahr) durch entsprechende Reaktion zu erledigen; sie gerät in die Neurose der Angst, wenn sie sich unfähig merkt, die endogen entstandene (Sexual-) Erregung auszugleiche. In https://www.projekt-gutenberg.org/ freud/kleine1/Kapitel7.html

8 Freud hat sich in Studien über Hysterie (Freud, 1895) mit dem Affekt befasst - sein Ursprung ist in einem traumatischen Erlebnis, das keine Abreagierung des Affektes erlaubt. Der Affekt ist also „blockiert“ und durch Aktivierung einer Erinnerung kann dieser Affekt nochmal erlebt und abgeführt wird - so verschwinden die Symptome. Es wurde von Freud und Breuer als Katharsis bezeichnet. Vgl. Döll-Hentschker, Susanne (2008): Psychoanalytische Affekttheorie(n) heute - eine historische Annäherung. In: Psychologie in Österreich 5, S. 446-447.

9 Freud, Sigmund (1946): Die Verdrängung. In: Gesammelte Werke, Band X. London: Imago, S. 255-256. 
In diesem Fall spricht man also von „Angst als umgewandelte Libido."10 Dazu führt Freud folgende Erklärung an: „Bei den Psychoneurosen sei zwar die Ursache für die Anhäufung unabgeführter Erregung eine psychische: die Verdrängung. Was freilich daraus folgt, sei das gleiche bei den Aktualneurosen: die angehäufte Erregung (oder Libido) ${ }^{11}$ verwandelt sich unmittelbar in Angst. ${ }^{12}$ Die Angst ist also ein Ergebnis des verwandelten Triebes.

Vor Freud wurde Angst als Reaktion auf eine Gefahrensituation oder eine traumatische Situation, die nach einem bestimmten Schema abläuft, aufgefasst. ${ }^{13}$ Sigmund Freud unterscheidet zuerst zwischen Realangst als Reaktion auf äußere Gefahren und neurotischer Angst:

Es haftet ihr [der Angst, V.B.] ein Charakter von Unbestimmtheit und Objektlosigkeit an; der korrekte Sprachgebrauch ändert selbst ihren Namen, wenn sie ein Objekt gefunden hat, und ersetzt ihn dann durch Furcht. Die Angst hat ferner außer ihrer Beziehung zur Gefahr eine andere zur Neurose, um deren Aufklärung wir uns seit langem bemühen. Es entsteht die Frage, warum nicht alle Angstreaktionen neurotisch sind, warum wir so viele als normal anerkennen; endlich verlangt der Unterschied von Realangst und neurotischer Angst nach gründlicher Würdigung. ${ }^{14}$

Die Konfrontation einer traumatischen Situation mit dem Erlebnis der Hilflosigkeit ist ein entscheidender Faktor für die automatische Angst. Gerade die Angst als Signal ist eine rationale Antwort des Ichs auf eine Gefahrensituation. Bei all den Gefahren ist Trennung von einem Liebesobjekt, Verlust des Liebesobjektes oder Verlust seiner Liebe ein gemeinsames Zeichen. Dies führt zur Ansammlung von unerfüllten Sehnsüchten und damit tritt ein Erlebnis der Hilfslosigkeit ein. ${ }^{15}$

Das Konzept von Angst als Signal (im Unterscheid zur automatischen Angst) „steht in enger Verbindung mit der Auffassung Freuds, dass das Denken die Affektentwicklung durch die Denktätigkeit auf jenes Minimum reduzieren müsse, welches für die Signalaus-

10 Gruber [Anm. 1], S. 229.

11 Bei Angstneurose und Hysterie „tritt an Stelle einer psychischen Verarbeitung eine Ablenkung der Erregung in das Somatische ein; der Unterschied liegt bloß darin, daß die Erregung, in deren Verschiebung sich die Neurose äußert, bei der Angstneurose eine rein somatische (die somatische Sexualerregung), bei der Hysterie eine psychische (durch Konflikt hervorgerufene) ist. Es kann daher nicht verwundern, daß Hysterie und Angstneurose sich gesetzmäßig miteinander kombinieren [und] daß die Hysterie eine Anzahl von Symptomen einfach der Angstneurose entlehnt“ (Studienausgabe VI: 49; 35-38). Daraus zog er den Schluss, daß die angehäufte Erregung in Angst umgewandelt werde [nicht natürlich in Libido mündete], d.h. Libido wird zu Angst. In: Ders (1999): Gesammelte Werke. Bd. XIV. Hg. von Anna Freud u. a. Frankfurt am Main: Fischer 1999, S. 138.

12 Ebd., S. 230.

13 Vgl. Ebd., S.230.

14 Freud, Sigmund (1994): Hemmung, Symptom und Angst. In: Studienausgabe, Band VI. Hysterie und Angst. Frankfurt am Main: Fischer, S. 45.

15 Vgl. Gruber [Anm. 1], S. 231. 
lösung unerlässlich ist. "16 Der Affekt wird in das Unbewusste als Angst abgeführt und die Angst produziert ein Signal, um einen Ausbruch stärkerer Angst zu vermeiden. ${ }^{17}$

\section{Angst vs. Trauma im Roman Erinnerungen eines Narren}

Die Wahl der Figuren geschieht oft - und Marianne Grubers Roman Erinnerungen eines Narren (2012) ist hier keine Ausnahme - im Kontext von psychologischen bzw. philosophischen Konzepten der Zeit, in der der Roman spielt. In der Wahl der historischen Epoche, in diesem Fall des zweiten Weltkriegs und der Nachkriegszeit, schlagen sich die Individualität des Autors, die Erwartungen des Lesepublikums bzw. des Literaturmarktes, der Blickwinkel auf die gewählte Epoche nieder. Die Rolle der Angst und des Traumas auf dem Weg, wie der Protagonist, ein Clown, Frieden mit der Welt zu schließen versucht, werden im Folgenden näher betrachtet.

Der Roman handelt von einem nur als Clown charakterisierten Mann, der dazu verurteilt ist, bewegungslos im Bett zu liegen, sich der verhassten Rehabilitation zu unterziehen, während er sich nach einem Zuhörer sehnt, der seine Rolle als Clown übernimmt. Er weiß von der mangelnden Kohärenz seiner Erinnerungen.

Der Text beginnt mit einer verfremdenden Abwandlung von Ludwig Wittgensteins Zitat. Statt eines Hinweises auf das Missverständnis der Logik unserer Sprache, die für metaphysische Themen untauglich ist („Was sich überhaupt sagen lässt, lässt sich klar sagen; und wovon man nicht reden kann, darüber muss man schweigen"18) lautet die Warnung des Clowns, der sich sein Leben lang nur durch die Körpersprache mit seinem Publikum verständigte, „man sollte nicht über Dinge sprechen, über die man nicht sprechen kann." ${ }^{19}$ Dies bezieht sich auf das Thema der Kommunikation, die in der ganzen Geschichte „fließt“20, denn der Protagonist bemüht sich, alles, was er erlebt hat, aus sich herauszuholen. Seine traumatischen Erlebnisse werden in nichts unterdrückt und lassen die Zuhörerin sogar erröten. Gleich am Anfang vergleicht der Clown seine Leere mit aussetzenden Erinnerungen - „Als ob man ausgeronnen wäre.“21 Es ist ein Versuch Lücken im Gedächtnis zu schließen, die nach Freud ein Trauma im Gedächtnis des Betroffenen hinterlässt. ${ }^{22}$ Es lässt sich nicht klar sagen und trotzdem will der vom Hochseil abgestürzte Clown nicht schweigen, obwohl sich das Erinnerte seiner Erzählkompetenz entzieht: „So viele Anfänge und kein Ende. So viele Enden und kein Anfang. Man muss

\footnotetext{
16 Ebd., S. 231.

17 Vgl. Ebd., S. 231.

18 Wittgenstein, Ludwig (2003): Tractatus logico-philosophicus. Frankfurt am Main: Suhrkamp, S. 7.

19 Gruber [Anm. 1], S. 5.

20 Gruber vergleicht Gewässer mit dem Strom der Rede. S. 5.

21 Gruber [Anm. 1], S. 5.

22 Vgl. Koch, Gertrud (1999): Bruchlinien. Tendenzen der Holocaustforschung. Köln - Weimar - Wien: Böhlau, S. 268.
} 
darüber reden, ehe es zu spät ist, wiewohl es immer zu spät ist oder zu früh, was auf das Gleiche hinausläuft.“"23

Laut Janoff-Bulmann gibt es zwei unterschiedliche Gruppen von Reaktionen auf traumatische Ereignisse, die sich nur scheinbar widersprechen. In der ersten Gruppe von Reaktionen geht es um die so genannte emotionelle Taubheit. Andererseits gibt es in der zweiten Gruppe aufgezwungene Gedanken zu jener Katastrophe und damit ein Wiedererleben des Traumas in allen Bewusstseinszuständen - auch in Tagträumen. Die Patienten schwanken zwischen dem Bedürfnis sich mit dem Trauma auseinanderzusetzen und dem Bedürfnis dieses Trauma zu verdrängen, um sich davor zu schützen. ${ }^{24}$ Der Protagonist des Romans beschreibt sein Leben, seine Kindheit, seine Lebensetappe im Zirkus - und damit erlebt er alles wieder. Der Wendepunkt seines Lebens ist der Tod seiner Mutter „und ihrem Tod folgte eine furchtbare Stille. “25 Neben dem Bedürfnis sein Trauma nochmal zu erleben, gibt es da auch Ansätze, um alles zu verdrängen. „Ich kann nicht. Nicht schweigen und nicht reden. ${ }^{\text {26 }}$

Nach dem Tod seiner Mutter hat sich alles verändert, und er ist durch ihren Tod zutiefst traumatisiert. Er scheiterte nicht nur die Kommunikation mit seinem Vater, sondern der Junge flüchtete auch vor der in der Schule offiziell verbreiteten Hoffnung, die ihm als „Gängelband der Mächtigen“27 vorkam. Zur Erklärung dieser Veränderung in der Lebensweise des Protagonisten ist anzumerken, dass die Folge der Traumatisierung eine geistige und emotionelle Erstarrung ist, die meistens bald nach dem traumatischen Ereignis anfängt. Die betroffene Person leidet an einem Gefühl der Isolation von Umgebung, Freunden oder Verwandtschaft. Es handelt sich um den Verlust des Interesses an der Tätigkeit, die diese Person früher ausübte..$^{28}$ Er verliert Interesse an seinem Studium, fühlt sich nach dem Tod der Mutter beim Vater nicht mehr zuhause, seine Einsamkeit treibt ihn zu kriminellen Taten, die eine anarchistische Motivation haben.

Er „stank nach Verwahrlosung und Elend“29, ohne seine Mutter vergessen zu können. Ein neues Zuhause fand der von Angst und Kälte geplagte Ausreißer in einem Zirkus, wo er ausüben musste, was die anderen Arbeiter nicht machen wollten. Die größte Demütigung war sein Gestank, der als Metapher für den verlorenen Lebenssinn gelesen werden kann. Die Arbeit und die Unterkunft bei dem Clown Hieronymo, ein neuer Lebenssinn, helfen ihm sich teilweise über seine traumatischen Erinnerungen an seine Mutter und seinen Vater hinweg zu setzen. Laut Brigitte Lueger-Schuster sind die Symptome der posttraumatischen Belastungsstörung merklich stärker, wenn man mit Aktivitäten und Situationen, die dem traumatischen Ereignis ähnlich sind und die Erinnerung daran

23 Gruber [Anm. 1], S. 9.

24 Vgl. Lueger-Schuster, Brigitte (1998): Klinische Psychologie und Gesundheitspsychologie: WUV Universitätsverlag, Wien, S. 102.

25 Gruber [Anm. 1], S. 21.

26 Gruber [Anm. 1], S. 31.

27 Gruber [Anm. 1], S. 296.

28 Vgl. Lueger-Schuster [Anm. 24], S. 104.

29 Gruber [Anm. 1], S. 34 
wachrufen, in Kontakt kommt. ${ }^{30}$ Egal, wo der Junge war oder was er machte: In Gedanken war er immer der Sohn, der (nicht nur) seine Mutter verloren hat.

Wie bereits im theoretischen Teil erklärt wurde, beschreibt Sigmund Freud die Angst als ein Ergebnis der Konfrontation zwischen einer traumatischen Situation und Hilflosigkeit. Es geht also um eine Reaktion der Psyche auf eine ausweglose Situation. Im Fall des Romans Erinnerungen eines Narren geht es gerade um den Verlust der Mutter, des Zuhauses, des Vaters und sich selbst - der Junge hat sogar seine Identität verloren und fand eine neue als Clown und Schüler Hieronymos. „Man kann vor der Angst nicht fliehen, weil man nicht weiß, wohin, weil wir sie von keinem Orte herkommend erleben." ${ }^{31}$ Wenn man also vor der Angst nicht fliehen kann, ist die Angst ein Bestandteil des Menschen, der nicht nur unvermeidbar ist, sondern auch während des Lebens den Charakter des Individuums formt. Diese Behauptung knüpft an die Angsttheorie Freuds an. Die Angst ist angeboren und reflektiert die Typologie des Menschencharakters.

Die Flucht des Jungen ist ein Signal dafür, dass er eine gewisse Distanz zu dem emotional belastenden Zuhause suchte. Dies hängt mit Riemanns Angst vor der Hingabe zusammen. Er bemühte sich unabhängig zu sein, aber gleichzeitig wollte er vor seiner Angst fliehen.

„Ich sollte nirgendwo landen, antwortete ich trotzig. Ich bin von einem Zug abgesprungen, weil er gerade langsam fuhr. Und da war das Zirkuszelt, das ist alles. ${ }^{\text {32 }}$ Der Besitzer des Zirkus schickt ihn zu Hieronymo in die Lehre. Als Clown bemüht er sich vor seiner Vergangenheit und von den traumatischen Erinnerungen zu fliehen und dabei flieht er auch vor sich selbst. Er verliert seine eigene Identität und spielt eine andere Rolle.

Die Frage lautet wohl, was uns zusammenhält. Alles. Kann sein. Oder nichts. Oder vielleicht doch wiederum nur diese Geschichten, die ich mir erzähle, die ich Ihnen erzähle, die der Andere bringt, um mein Gedächtnis zu trainieren, die ich erfinde und missdeute, bis mein eifriges Fleisch zerfällt. ${ }^{33}$

Die Erinnerungen und das Geschichtenerzählen formen das Individuum und das Kollektiv. Auf Erinnerungen bauen wir unser Leben, unser Selbstverständnis und letztendlich auch die Wahrnehmung des historischen und aktuellen Kontextes auf. Die Erinnerung bildet die Substanz für die Konstruktion der individuellen und kollektiven Identität. ${ }^{34}$ Da das Gedächtnis ein unmittelbarer Bestandteil des Individuums ist, und zwar auch in dem Fall, wenn man Angst hat, dass seine (traumatischen) Erinnerungen zerfallen. Die Flucht aus dem Internat geschieht, als zu dem familiären Desaster noch die drückende politische Lage im Lande hinzukommt: „Ich habe geschwiegen. Es fiel mir nicht schwer.

30 Vgl. Lueger-Schuster [Anm. 24], S. 104.

31 Koch, Lars (2013): Angst. Ein interdisziplinäres Handbuch. Stuttgart: J.B. Metzler, S. 43.

32 Gruber [Anm. 1], S. 40.

33 Gruber [Anm. 1], S. 59.

34 Vgl. Kratochvil, Alexander (2016) (hrsg.): Pamět a trauma pohledem humanitních věd: Akropolis, Praha, S. 5-11. 
Das Schweigen war nach Mutters Tod und durch mein Internatsleben sozusagen zu meiner zweiten Natur geworden. Ich wusste: Wer redet, ist verloren. Das wir auch so verloren sind, begriff ich damals noch nicht." 35

Falls man nach einer schmerzvollen Situation natürlich schweigt, will man die traumatische Situation auf diese Art und Weise bewusst vermeiden ${ }^{36}$ und dabei seine Emotionen unterdrücken. Das Leben im Zirkus war für den Protagonisten eine Art der Isolation von der Realität. Er war im Zirkus versteckt, hat sein neues Leben unter der Maske eines Clowns ${ }^{37}$ gelebt und suchte so den Sinn des Lebens, indem er und Hieronymo Flüchtlinge retten. ${ }^{38}$

Der Zirkus war sein neues Zuhause, diese Distanz zu seinem alten Leben war für ihn sicher. Er verliebte sich in Rachel, fand einen Freund (Rollo) und einen Ersatzvater (Hieronymo). „Das Leben ist eine einzige Kette von Variationen des immer Gleichen."39 Nach und nach verlor er aber seine Liebe und seine Freunde, mit dem Absturz vom Hochseil entsteht ein neues Bedürfnis die Angst zu überwinden. Es ist keine Angst vor dem Tod. Traumatische Erlebnisse müssen als Worte entlastet werden. Der Protagonist liegt bewegungslos in seinen Bett und erzählt seine Geschichten.

„Über den Rest nicht viele Worte, je länger das Leben dauert, desto weniger gibt es zu berichten, alles drängt sich zusammen." ${ }^{40}$

\section{Der Clown als absurder Mensch}

Die Götter hatten Sisyphos dazu verurteilt einen Felsblock unablässig den Berg hinaufzuwälzen, von dessen Gipfel der Stein kraft seines eigenen Gewichts wieder hinunterrollte. Sie meinten nicht ganz ohne Grund, es gäbe keine grausamere Strafe, als unnütze und aussichtslose Arbeit. ${ }^{41}$

Der Tod der Mutter des Protagonisten in Grubers Roman ist der Beginn einer Phase, in der der kleine Junge zum Obdachlosen, zum Flüchtling, dann zum Clown und schließlich zum bettlägerigen Mann wird. Obwohl es schwierige Lebensphasen sind, gibt der Protagonist bis zum letzten Moment nicht auf. Er akzeptiert immer seine Situation und versucht, das Beste daraus zu machen. So wie nach dem Tod seiner Mutter, als er versuchte, mit seinem Vater in Kontakt zu treten und ihm die Geschichte von Sisyphos erzählte, die er ein wenig verbesserte. Heinzl weist darauf hin, dass der Protagonist seinen

35 Gruber [Anm. 1], S. 77.

36 Koch [wie Anm. 32], S. 123.

37 Gruber [Anm. 1]: „Hinter der Maske... war nichts an mir ich.“ S. 89.

38 Gruber [Anm. 1], S. 130.

39 Gruber [Anm. 1], S. 308.

40 Gruber [Anm. 1], S. 292.

41 Camus, Albert (2003): Der Mythos des Sisyphos. Reinbek bei Hamburg: Rowohlt, S.155. 
eigenen Mythos geschaffen hat, der dem Unsinn einen Sinn gibt. ${ }^{42}$ Ist dies eine Anspielung auf Camus' Sisyphos? „Sisyphos amüsiert sich vermutlich. Und wahrscheinlich paßt es Zeus nicht, daß sich sein Kontrahent amüsiert. Darf ihm nicht passen. Ein lachender Sisyphos, das fehlte gerade. Das Gelächter ist Zeus’ Monopol.“43

Camus nimmt den mythologischen Stoff auf und verbindet ihn mit seinem Werk, indem er Sisyphos als einen absurden Menschen begreift, der sich damit abgefunden hat, dass er seinen Stein endlos rollen wird - es ist für ihn keine Strafe mehr, sondern eine Aufgabe. ${ }^{44}$ Bei Gruber wie bei Camus geht es darum, dass man sich befreit, indem man den Sinn einer Situation erkennt, auch wenn sie absurd erscheint.

Das heißt, selbst wenn man sich in einer ausweglosen Situation befindet (wie der Protagonist des Romans, der traumatisiert und voller Angst ist), kann man sich retten. Zunächst dank Hieronymo, der ihm half, diesen Sinn zu finden, dann aber auch aus eigenem Antrieb, als er in seinen Erinnerungen zu dem zurückkehrte, was ihm noch Sinn gab, um weiterzuleben.

\section{Conclusio}

Das Motiv der Angst taucht in Grubers Roman Erinnerungen eines Narren in mehreren Transformationen auf. Da die Geschichte retrospektiv erzählt wird, lernt man die Angst zunächst als einen Bestandteil des Lebens und des darauffolgenden Sterbens kennen. Der Protagonist fürchtet sich nicht mehr und erwartet eine große Stille. ${ }^{45}$

Die Angst ist zwar ein unmittelbarer Bestandteil der menschlichen Existenz und hat die Fähigkeit, sogar in den menschlichen Erinnerungen in verschiedenen Formen stekken zu bleiben. Es geht nicht nur um das ängstliche Gefühl, sondern auch um Bilder oder Gerüche, die die Fähigkeit haben, die Angst bei dem Menschen wieder zu erregen. ${ }^{46}$ „Den Geschmack der Angst werden wir uns nie mehr aus dem Mund waschen können, sagte Rollo.“ ${ }^{47}$ „Nicht der Clown, den Hieronymo dafür lobt immer das Leben im Blick zu haben." ${ }^{8}$

Die andere Gestalt der Angst, ist die unerklärliche Angst. Der Protagonist kann die Angst offenbar nicht begründen, sie ist aber trotzdem präsent. Es ist die Angst vor dem Vergessen und Verlust seines Zuhauses. ${ }^{49}$

Bei der Aufdeckung der Formen von Angst in Grubers Roman stellt sich die Frage, ob und wie es möglich ist, mit Angst umzugehen. Es gibt mehrere Handlungsebenen, die

\footnotetext{
42 Vgl. Polt-Heinzl [Anm. 2].

43 Gruber [Anm. 1], S. 75.

44 Vgl. Camus [Anm. 42], S.159.

45 Gruber [Anm. 1], S. 6.

46 Vgl. Gruber [Anm. 1], S. 21.

47 Gruber [Anm. 1], S. 234.

48 Gruber [Anm. 1], S.234.

49 Vgl. Gruber [Anm. 1], S. 270.
} 
sich durch den Roman ziehen, aus denen die Sisyphos-Erzählung von Vater und Sohn hervorsticht, die verändert wurde, vielleicht nur, um dem Leser Camus' Konzept des Absurden näher zu bringen. Laut Camus muss man sich „Sisyphos glücklich vorstellen“50, das heißt, dass er sich mit der Tatsache abgefunden hat, dass er sinnlos einen Stein vor sich her rollt, der irgendwann ohnehin wegrollen wird.

Obwohl Polt-Heinzl das Ende des Romans mit Pessimismus durchsetzt und darauf hinweist, dass der Protagonist den Staffelstab weitergibt, um weiter eine Lüge zu leben, ${ }^{51}$ ist dies nicht die einzige mögliche Interpretation. Wenn der Clown als ein absurder Mensch im Camus'schen Sinn verstanden wird, ist er nicht unglücklich. Der Roman beschreibt seine wirkliche Lebensgeschichte, gibt sie an einen imaginären Zuhörer weiter und beweist damit, dass er sein Leben so akzeptiert hat, wie es war.

\section{Quellen und Literatur}

\section{Primärquelle}

Gruber, Marianne (2012): Erinnerungen eines Narren. Innsbruck - Wien: Haymon.

\section{Literatur}

Camus, Albert (2003): Der Mythos des Sisyphos. Reinbek bei Hamburg: Rowohlt.

Döll-Hentschker, Susanne (2008): Psychoanalytische Affekttheorie(n) heute - eine historische Annäherung. In: Psychologie in Österreich 5, S. 446-447.

Frankl, Viktor E. (1992): Man's Search for Meaning. An introduction to logotherapy. Fourth Edition. Freud, Sigmund (1999): Gesammelte Werke. Bd. I. Frankfurt am Main: Fischer.

Freud, Sigmund (1946): Die Verdrängung. In: Gesammelte Werke, Band X. London: Imago, S. $255-256$.

Freud, Sigmund (1994): Hemmung, Symptom und Angst. In: Studienausgabe, Band VI. Hysterie und Angst. Frankfurt am Main: Fischer.

Freud, Sigmund (1999): Gesammelte Werke. Bd. XIV. Hg. von Anna Freud u. a. Frankfurt am Main: Fischer.

Gruber, Marianne (1977): Existenzanalyse und Literatur. In: Podium 26, S. 19-23.

Koch, Lars (2013) (hrsg.): Angst. Ein interdisziplinäres Handbuch. Stuttgart: J.B. Metzler.

Kratochvil, Alexander (2016) (hrsg.): Pamět a trauma pohledem humanitních věd: Akropolis, Praha.

Lueger-Schuster, Brigitte (1998): Klinische Psychologie und Gesundheitspsychologie: WUV Universitätsverlag, Wien.

Polt-Heinzl, Evelyne (2012): Der Clown als stummer Zeuge. In: Die Presse, 15.06.2012 [online]. [Zit. 15.11.2021]. Erreichbar unter: https://www.diepresse.com/766285/der-clown-als-stummer-zeuge

50 Camus [Anm. 42], S. 145.

51 Vgl. Polt-Heinzl [Anm. 2]. 
Weidenhiller, Ute (1995): Ein Gespräch mit Marianne Gruber am 8. August 1995 in Steindorf am Ossiachersee in Marianne Gruber und die österreichische Gegenwartsliteratur. Die Wille zum Sinn in einer sinnlosen Welt, 1995.

Wittgenstein, Ludwig (2003): Tractatus logico-philosophicus. Frankfurt am Main: Suhrkamp.

Mgr. Václava Beyerová / vaclava.beyerova@mail.muni.cz

Masarykova univerzita, Filozofická fakulta, Ústav germanistiky, nordistiky a nederlandistiky Arna Nováka 1, 60200 Brno, CZ 
